\title{
DETERMINATION OF THE COMPLETE ODF OF CUBIC SYSTEM MATERIALS BY THE MAXIMUM ENTROPY METHOD
}

\author{
WANG, FU, XU, JIAZHENG AND LIANG, ZHIDE \\ Department of Materials Science and Engineering, Northeast University of \\ Technology, Shenyang, Liaoning 110006, China
}

(10 September, 1990)

\begin{abstract}
A maximum entropy method (MEM) suitable for determining the complete ODF of cubic system materials is developed. This method treats the cubic system material texture data just as if they were if tegragonal besides introducing the relations among $W \operatorname{lmn}$ which characterize the cubic system. As experimental evidence this method has been applied to determine the complete ODF of a cold-rolled deep-drawing steel sheet sample and the result shows that the texture of the sample, as is supposed, consists of a $\{111\}$ fiber and an incomplete $\langle 110\rangle$ fiber only while the negative regions and the weak ghosts presented in the incomplete ODF of the same sample are disappeared here.
\end{abstract}

KEY WORDS ODF calculation, Maximum entropy method, Cubic crystal symmetry, Rolling texture of iron.

\section{INTRODUCTION}

The maximum entropy method (MEM) provides the most probable solution of problems with insufficient information, so it ought to be suitable for the complete ODF determination. This method was at first successfully applied to the determination of the inverse pole figure of hexagonal system material in 1987 and its good reliability was proved by means of model simulation as well (Wang et al. 1987). Then the complete ODF determination of hexagonal system material by the MEM was also achieved (Wang et al. 1989). Here the determination of the complete ODF of cubic system material by the MEM is made, which is basically the same as for the tetragonal system material besides introducing the relations among $W \operatorname{lmn}$ which characterize the cubic system.

\section{PRINCIPLE}

Suppose there are three sample symmetry planes each perpendicular to one of the sample coordinate axes, three crystal symmetry planes each perpendicular to one of the crystallographic axes and a 4-fold symmetry axis coincides with the $C$ axis in addition, then the dimension of the asymmetric sub-orientation space adopted is $\psi, \theta, \phi=0^{\circ}-90^{\circ}$. Divide this space into $J$ orientation elements $(\sin \theta \Delta \theta \Delta \psi \Delta \phi)$ and let $V_{J}$ be the sample volume fraction oriented within 
element $j$, then the relation between the average orientation density $\omega\left(\theta_{J}, \psi_{J}, \phi_{J}\right)$ and $V_{J}$ takes the form

$$
V_{J}=32 \omega\left(\theta_{J}, \psi_{J}, \phi_{J}\right) \sin \theta_{J} \Delta \theta \Delta \psi \Delta \phi
$$

and

$$
\sum_{j=1}^{J} V_{J}=1 .
$$

Express the incomplete ODF series of the sample as for the tetragonal system, truncate the series at $\mathrm{L} \max$ and let $R$ be the total number of the non-zero $W \operatorname{lmn}$, however owing to the sample being really cubic there are some relations (Roe 1966) existing among $W \operatorname{lmn}$ which may be used to derive all the $R$ values from those known $W$ lmn. Now $W$ lmn holds (Wang et al. 1989)

$$
W_{r}=\sum_{j=1}^{J} A_{r J} V_{J}, \quad r=1,2, \ldots, R
$$

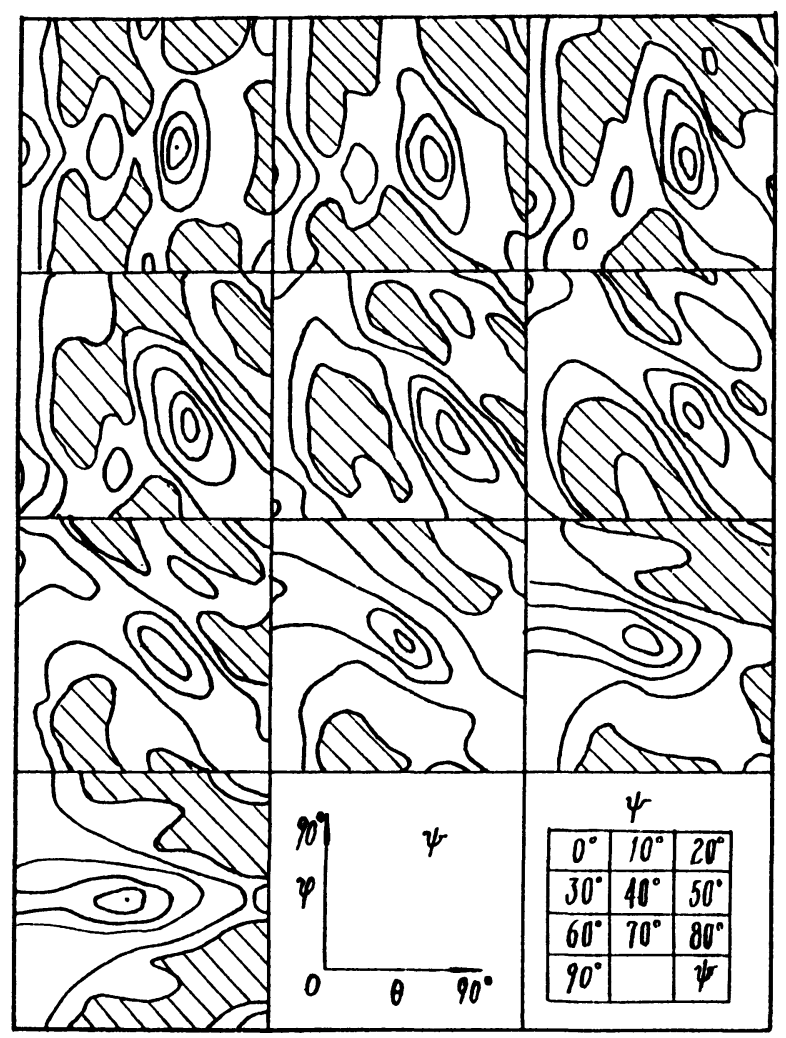

Figure 1 The incomplete ODF of a cold-rolled deep-drawing steel sheet (after H. Hu 1986). Levels, $1,3,5,7$. Hatched-negative region. 


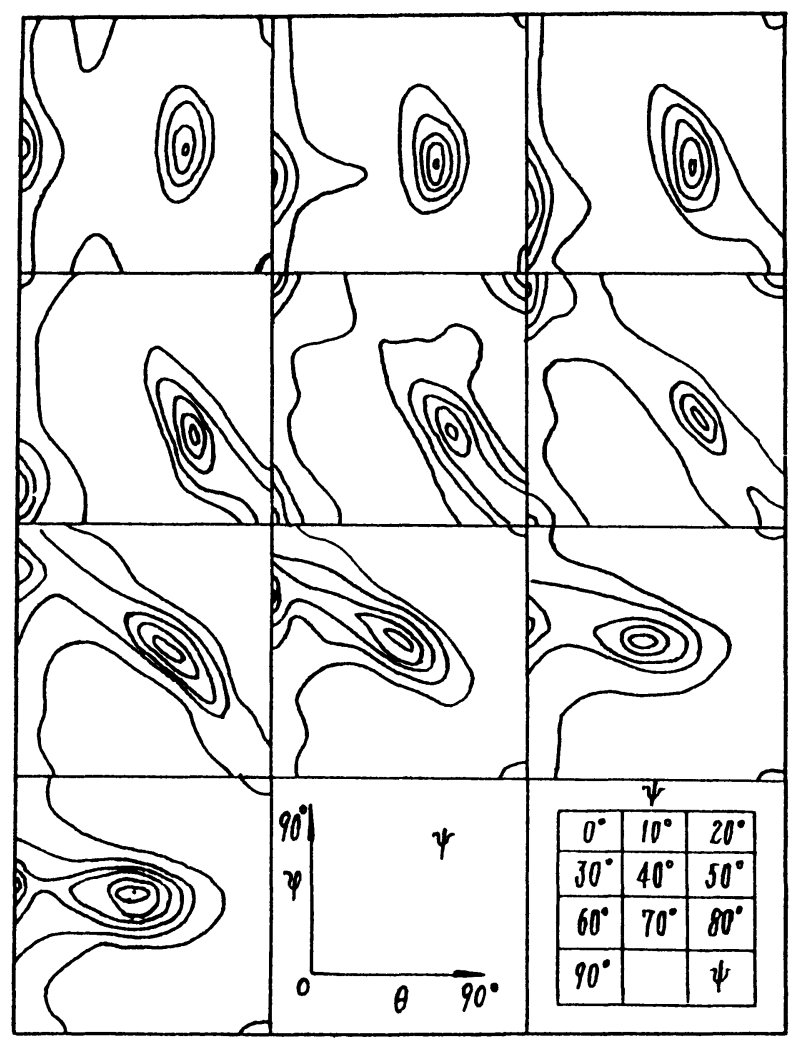

Figure 2 The complete ODF derived from the same sample by the MEM. Levels, 1, 3, 5, 7, 9, 11.

and

$$
A_{r J}=\frac{1}{8 \pi^{2}}\left\{Z \operatorname{lmn}\left(\cos \theta_{J}\right) \cos \left(m \psi_{J}+n \phi_{J}\right)+(-1)^{l} Z \operatorname{lmn}\left(\cos \theta_{J}\right) \cos \left(m \psi_{J}-n \phi_{J}\right)\right\}
$$

where $r$ is the order number of the $\operatorname{lmn}$ array; $Z \operatorname{lmn}(\cos \theta)$ are the augmented Jacobi polynomials. Following the MEM $V_{J}$ adopts the form

$$
V_{J}=\exp \left(-1-\lambda_{0}-\sum_{r=1}^{R} \lambda_{r} A_{r J}\right), \quad j=1,2, \ldots, J
$$

Here $\lambda_{0}, \lambda_{1}, \lambda_{2}, \ldots, \lambda_{R}$ are the unknown Lagrangian multipliers (Jaynes 1957). Introducing equation set (4) into equation set (2), a set of non-linear equations of these multipliers are obtained and all the multipliers can be solved by the modified Newton method or others. Finally utilizing Eq. (1) the complete ODF of the sample is obtained.

\section{EXAMPLE}

As an examination of this method a complete ODF (Figure 2) of a cold-rolled deep-drawing steel sheet was derived from its incomplete ODF (Figure 1) which 
was determined by $\mathrm{H}$. $\mathrm{Hu}$ (1986). There the incomplete ODF series was truncated at $L \max =16$ with $R=152$ while only 58 of them were independent and known. The sub-orientation space was divided into $J=5832$ orientation elements with $\Delta \psi=\Delta \theta=\Delta \phi=5^{\circ}$. Comparing Figure 2 with Figure 1 it is found that their configurations are like each other but the former appears more dense with peak level over 12 and no negative region while the later contains massive negative regions with valley level near -1.8 and the peak level is about 8 only. Moreover, the texture of the sample corresponding to the complete ODF, as is supposed, is just composed of a $\{111\}$ fiber and an incomplete $\langle 110\rangle$ fiber while the weak peaks which appeared merely in the incomplete ODF are not consistent with rational texture components. Hence it may be considered that the complete ODF derived by the MEM is rather reliable.

Incidently the authors have been advised to test the MEM by directly measuring the orientations of crystallites of a sample one by one (Morris 1989). Hence, the crystallite orientations of an electrical silicon steel sheet containing 308 crystallites have been measured accurately by a modified etch pit method. Surely this was not enough to examine the complete ODF determination with such a few crystallite orientations (Wagner 1986), but it could be verified (Wang et al. 1990) that the inverse pole figure determination by the MEM was excellent.

\section{CONCLUSIONS}

The MEM developed for determining the complete ODF of cubic system materials is suitable for use and its reliability is confirmed by experiment test.

\section{References}

Hu, H. (1980). Acta Metall. Sinica 16, 121.

Jaynes, E. T. (1957). Phys. Rev. 106, 620.

Morris, P. R. (1989). Private Communication. Roe, R. J. (1966). J. Appl. Phys. 37, 2068.

Wagner, F. (1986). Experimental Techniques of Texture Analysis. Ed. H. J. Bunge. DGM. Informationsgesellschaft, Oberursel. p. 115.

Wang, F., Xu, J. and Liang, Z. (1987) Proc. JCOTOM-8. The Metallurgical Society of AIME. Warreudale. p. 111.

Wang, F., Xu, J. and Lang, Z. (1989). Textures and Microstructures 10, 217.

Wang, F. Xu, J. and Liang, Z. (1990) J. Appl. Cryst. (in press). 